Revista de BiOlOgía Tropical

\title{
La estructura de tallas como evidencia del establecimiento de Pterois volitans (Scorpaeniformes: Scorpaenidae) en el sur del Caribe mexicano
}

\author{
Miguel Mateo Sabido-Itzá ${ }^{1}$, Alejandro Medina-Quej ${ }^{2}$, Alberto de Jesús-Navarrete ${ }^{3}$, Jorge Manuel \\ Gómez-Poot ${ }^{4} \&$ María del Carmen García-Rivas ${ }^{4}$ \\ 1. Maestría en Manejo de Zona Costera. Instituto Tecnológico de Chetumal, Av. Insurgentes No. 330, C.P. 77013, Col. \\ David Gustavo Gutiérrez, Chetumal, Quintana Roo, México; mateosabido@gmail.com \\ 2. Depto. de Química y Bioquímica. Instituto Tecnológico de Chetumal, México; lexobu@hotmail.com \\ 3. Depto. de Sistemática y Ecología Acuática. El Colegio de la Frontera SUR-Unidad Chetumal, México; \\ anavarre@ecosur.mx \\ 4. Parque Nacional Arrecifes de Xcalak. Comisión Nacional de Áreas Naturales Protegidas, México; \\ jmgomez74@msn.com,mcgarcia@conanp.gob.mx
}

Recibido 29-IV-2014. Corregido 10-X-2015. Aceptado 03-XI-2015.

\begin{abstract}
Size structure as evidence of population establishment of Pterois volitans (Scorpaeniformes: Scorpaenidae) in the South Mexican Caribbean. The lionfish ( $P$. volitans) has now invaded all the Mexican Caribbean and Gulf of Mexico, with the potential to cause negative impacts on the reefs. In the South Mexican Caribbean was firstly reported in July 2009, and six years after this report, some control measures such as fish tournament and local marketing have been implemented. However, information on its biology and invasion is still-lacking, so this study analyzed the population structure of 2164 organisms collected from 2009 to 2012. An increase was observed in sizes for each year averaging Total length $(T l): 118 \pm 34.8,133 \pm 56.3,187 \pm 74.8$ and $219 \pm 72.4 \mathrm{~mm}$, respectively. Lionfish establishment at the study site is shown for the presence of juveniles' sizes $20 \mathrm{~mm}$ TL up to $375 \mathrm{~mm}$ TL. When the back-calculation was obtained, we estimated that the larger fish could have recruited in early 2006, three years before the first report was made. A continuous population monitoring and an ecological study, will allow us to clarify the real impact in the ecosystems of the region and so to propose the most effective control actions. Rev. Biol. Trop. 64 (1): 353-362. Epub 2016 March 01.
\end{abstract}

Key words: Lionfish, invasive species, Mexican Caribbean, size structure.

A nivel mundial, las invasiones biológicas y la destrucción del hábitat, son las amenazas más claras a la biodiversidad, la conservación de los ecosistemas y sus servicios ambientales (IMTA, Conabio, GECI, Aridamérica, \& TNC, 2007).

Las especies exóticas son aquellas presentes en áreas fuera de su distribución natural y cuyas características les confieren la capacidad de colonizar, establecerse y causar daños significativos al ambiente (Edelist, Rilov, Golani, Carlton, \& Spanier, 2013; IMTA et al., 2007). El pez león ( $P$. volitans y $P$. miles) originario del Indo-Pacífico (Schultz, 1986), es el primer pez invasor en establecerse en el oeste del Atlántico Norte (Schofield, 2009, 2010).

Desde su introducción accidental en el Atlántico en los años 80's (Semmens, Buhle, Salomon, \& Pattengill-Semmens, 2004), P. volitans ha ampliado su distribución prácticamente en todo el Caribe, ya que las condiciones ambientales suelen ser mejores que las de su hábitat original (Darling, Green, O'Leary, \& Côté, 2011). Además de representar una invasión rápida (Ruttenberg et al., 2012), según Sutherland et al. (2010), la considera entre los problemas que más afectan la conservación de la diversidad biológica en el Caribe. Esta 
especie alcanza mayores tallas y densidades poblacionales en sus nuevas áreas, que en las nativas debido a su alta tasa reproductiva (Gardner et al., 2015), a la ausencia de depredadores (Darling et al., 2011), a su habilidad competitiva con especies nativas (Albins, 2013) y al apetito voraz, siendo un consumidor generalista (Valdez-Moreno, Quintal-Lizama, Gómez-Lozano, \& García-Rivas, 2012). Los impactos derivados de su presencia en los arrecifes del Atlántico van desde la reducción en el reclutamiento de peces hasta un $79 \%$ y una disminución en la biomasa de $65 \%$ (Albins \& Hixon, 2008; Green, Akins, Maljković, \& Côté, 2012). Además, los efectos directos e indirectos de $P$. volitans se podrían combinar con otros factores de estrés como la sobre pesca y alteraciones de la comunidad arrecifal (Albins \& Hixon, 2013).

El primer reporte de su presencia en aguas mexicanas, ocurrió en enero del 2009 en los arrecifes de Cozumel (Schofield, 2009), posteriormente en 2010 se reportó la captura de un ejemplar en los Arrecifes de Alacranes en el Golfo de México en Yucatán (Aguilar-Perera \& Tuz-Sulub, 2010); y en 2012 (Santander-Monsalvo, López-Huerta, Aguilar-Perera, \& TuzSulub, 2012), se registró en el Parque Nacional Sistema Arrecifal Veracruzano (PNSAV) (Santander-Monsalvo et al., 2012).

En el Parque Nacional Arrecifes de Xcalak (PNAX), se capturó por vez primera en julio del 2009. A partir de esta fecha se ha incrementado el número de avistamientos y capturas en diferentes zonas dentro del área protegida. No obstante, los estudios sobre $P$. volitans son escasos para la parte del Caribe mexicano. Debido a esto, el presente estudio tiene como finalidad proveer de información básica para el entendimiento de esta especie, puesto que aún no son claros los impactos sobre las comunidades marinas de la zona. También se argumenta que la existencia de una estructura de tallas y pesos altos, indican un establecimiento poblacional, ya que esta pudo ocurrir antes del primer avistamiento.

\section{MATERIALES Y MÉTODOS}

Área de estudio y colectas en campo: El Parque Nacional Arrecifes de Xcalak (PNAX), se localiza en la costa sur del estado de Quintana Roo, México, tiene una superficie de 17 949.456 ha, de las cuales 13495 ha corresponden a ecosistemas marinos y 4543 ha a humedales, que incluyeron lagunas permanentes y temporales (CONANP, 2004). Esta Área Natural Protegida forma parte del Sistema Arrecifal Mesoamericano (SAM), considerado como sitio prioritario de la biodiversidad (Fig. 1) (Kramer \& Kramer, 2002).

Los peces fueron recolectados dentro del polígono del PNAX a partir de la primera captura registrada el 23 de julio 2009, en el sitio llamado "Portillas" a una profundidad de un $\mathrm{m}$. (18 13 '53.70” N - 8750’21.90” W), con una $L t$ de $70 \mathrm{~mm}$ y peso de $5 \mathrm{~g}$, y hasta el año 2012 . Los peces fueron capturados por pescadores, pobladores, guarda parques y voluntarios, utilizando redes de mano y arpones, durante salidas rutinarias de personal del PNAX, pesca incidental y dentro del Programa de Empleo Temporal (subsidio federal).

Se utilizó snorkel y equipo SCUBA, con visitas a diversas zonas a profundidades entre 0.5 y $57 \mathrm{~m}$. Para georreferenciar los sitios de captura de organismos se utilizó un GPS marca Garmin.

Trabajo de laboratorio: A cada organismo capturado se le asignó un número de serie incluyendo información relacionada con: fecha de captura, lugar, profundidad, se midió la longitud total $(L t)$, mediante un ictiómetro convencional $(\mathrm{mm})$ y el peso total con una báscula digital con precisión de $1 \mathrm{~g}$.

Se utilizó el test no paramétrico de Kruskal-Wallis, para comparar anualmente las tallas de los organismos capturados y establecer si había o no diferencias significativas en estas. Así mismo, para describir el cambio interanual de la composición de tallas, se utilizaron las frecuencias relativas por intervalos de 10 $\mathrm{mm}$ de $L t$. Se compararon las distribuciones 


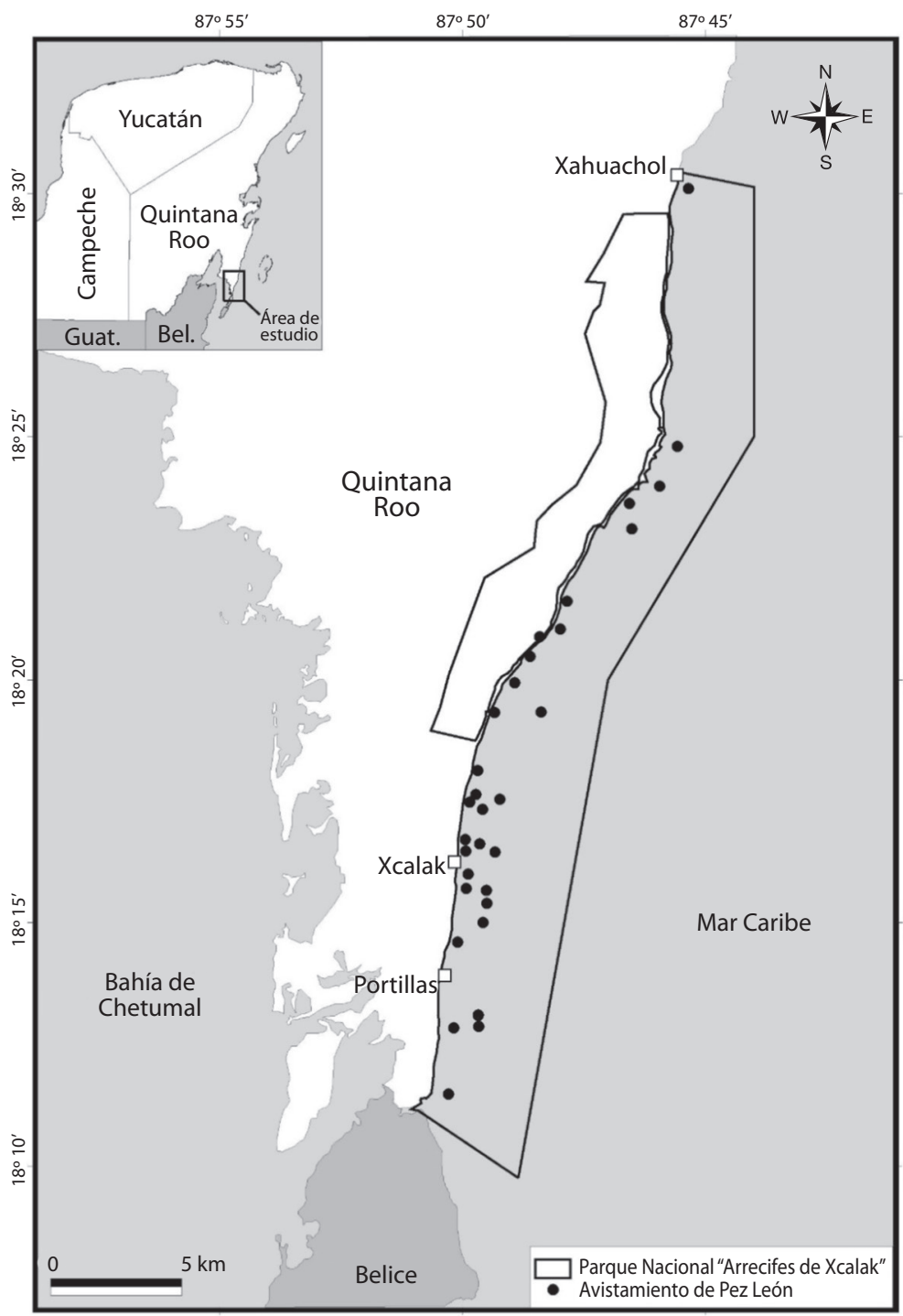

Fig. 1. Distribución de los avistamientos y capturas de pez león dentro del polígono del Parque Nacional Arrecifes de Xcalak, México.

Fig. 1. Distribution of sightings and catches of lionfish inside the polygon Parque Nacional Arrecifes de Xcalak, México.

anuales de tallas con la prueba no paramétrica Kolmogorov-Smirnov.

La relación longitud-peso fue representada por la ecuación (Ricker, 2010):

$$
\mathrm{W}=\mathrm{a} * \mathrm{~L}^{\mathrm{b}}
$$

Donde $(W)$ es el peso, $(a)$ coeficiente de condición, $(L)$ la longitud y $(b)$ pendiente. Para determinar si el crecimiento fue alométrico o isométrico se utilizó el valor del exponente $b$ mediante una prueba t-student, comparando con el valor de 3.0 que corresponde a un crecimiento isométrico.

La edad fue calculada a partir de la $L t$; utilizando la ecuación de crecimiento de Von Bertalanffy, (1957):

$$
L t=L_{\infty}\left(1-\mathrm{e}^{-\mathrm{k}(\mathrm{t}-\mathrm{t})}\right)
$$


Dónde: $(L t)$ longitud en tiempo t, $\left(L_{\infty}\right)$ longitud máxima que puede alcanzar el pez. $(k)$ es la tasa de crecimiento (medida de la velocidad a la cual la longitud se aproxima a la $L_{\infty)}$ y $\left(\mathrm{t}_{0}\right)$ momento en que la longitud es igual a 0 .

Para estimar la fecha inicial de la invasión en el sur del Caribe mexicano y la edad promedio de los organismos capturados, se obtuvieron los retrocálculos de cada año de muestreo. Se utilizó la información aportada por Edwards, Frazer y Jacoby (2014) para la población de Little Cayman en el Caribe, relativa a: $\left(L_{\infty}=382 \mathrm{~mm}\right),(k=0.38)$ y $\left(\mathrm{t}_{0}=-1.01\right)$.

\section{RESULTADOS}

Se obtuvieron 2164 registros de pez león con rango de tallas entre 25 a $375 \mathrm{~mm}$ de $L t$, $(163.8 \pm 72.4 \mathrm{~mm})$ y con peso que osciló entre $0.5 \mathrm{~g}$ a $859.0 \mathrm{~g},(101.3 \pm 128.8 \mathrm{~g})$ (Cuadro 1$)$.

En 2009 se obtuvieron 36 registros, como consecuencia de la reciente invasión en Xcalak. No obstante, se observó aumento en las tallas y pesos promedio por año; en 2009, 2010 y 2011 se observó un aumento en las tallas y pesos promedios y un incremento en la Lt máxima por año (Cuadro 1). En 2012, se presentó el menor número de capturas $(\mathrm{N}=21)$, tomándose datos de hasta febrero. Mediante la prueba de Kruskal-Wallis, se halló que las tallas fueron significativamente diferentes durante los años de muestreo; (K-W, P < 0.05). Sin embargo, entre 2009 y 2010, no se encontraron diferencias significativas $(\mathrm{P}>0.05)$.

Para el 2009, se evidenció un pico de abundancia a los $110 \mathrm{~mm}$ de $L t$. En 2010, la distribución fue binomial con picos entre 80 y $129 \mathrm{~mm}$ de Lt. Durante el 2011 se formaron dos picos nuevamente, el primero ubicado entre 80 y $109 \mathrm{~mm}$ de $L t$ y el segundo a partir de los 180 y $209 \mathrm{~mm}$ de Lt. A inicios del 2012, se observó un desplazamiento de los dos principales grupos modales a intervalos de mayor longitud comprendiendo de 150 a 229 y de 290 a $329 \mathrm{~mm} \mathrm{Lt} \mathrm{(Fig.} \mathrm{2).} \mathrm{Al} \mathrm{agrupar} \mathrm{el} \mathrm{total} \mathrm{de} \mathrm{las}$ muestras se puede observar un grupo modal claramente definido por las principales tallas capturadas oscilando entre 80 a $169 \mathrm{~mm}$ de Lt. Se detectaron diferencias significativas en la estructura de tallas por año (K-S, P < 0.05), a excepción de la comparación entre 2009 y $2010(\mathrm{P}>0.05)$.

La relación entre la $L t$ y el peso de $P$. volitans se calculó a partir de 2143 peces que tuvieron registros completos de $L t$ y peso. La curva de mejor se ajusta fue de tipo potencial, con un coeficiente de correlación $r^{2}=0.9907$ (Fig. 3). Debido a que el valor de la pendiente fue $\mathrm{b}=3.1832$, se consideró que el tipo de crecimiento es alométrico; la prueba estadística t-student, $\left(\mathrm{t}_{\mathrm{s}}=5.6446 \mathrm{E}-80, \mathrm{P}<0.05\right)$ confirmó el supuesto. Asimismo, se realizó

CUADRO 1

Longitud total y peso promedio $( \pm \mathrm{DE})$, mínimo, máximo y número de muestras por año de captura del pez león (Pterois volitans) en el Parque Nacional Arrecifes de Xcalak, Q. Roo, México

TABLE 1

Mean Total length and weight $( \pm \mathrm{SD})$, minimum, maximun and number of samples by catch year off Lionfish (Pterois volitans) in Parque Nacional Arrecifes de Xcalak, Q. Roo, Mexico

\begin{tabular}{cccccccc} 
& \multicolumn{3}{c}{ Media } & \multicolumn{2}{c}{ Mínimo } & \multicolumn{2}{c}{ Máximo } \\
Año & LT $(\mathrm{mm})$ & Peso $(\mathrm{g})$ & LT $(\mathrm{mm})$ & Peso $(\mathrm{g})$ & LT $(\mathrm{mm})$ & Peso $(\mathrm{g})$ & \\
2009 & $118.8 \pm 34.8$ & $31.1 \pm 32.0$ & 40 & 1 & 210 & 126 & 36 \\
2010 & $133.1 \pm 56.3$ & $51.1 \pm 70.2$ & 25 & 0.5 & 321 & 528 & 915 \\
2011 & $187.84 \pm 74.8$ & $142.2 \pm 149.3$ & 38 & 0.5 & 375 & 859 & 1192 \\
2012 & $219.7 \pm 55.9$ & $\mathrm{ND}$ & 125 & $\mathrm{ND}$ & 324 & $\mathrm{ND}$ & 21 \\
Total & $163.84 \pm 72.44$ & $101.35 \pm 128.84$ & 0.5 & 375 & 375 & 859 & 2164 \\
\hline
\end{tabular}

$\mathrm{ND}=$ No Datos.

$\mathrm{DN}=$ No Data. 

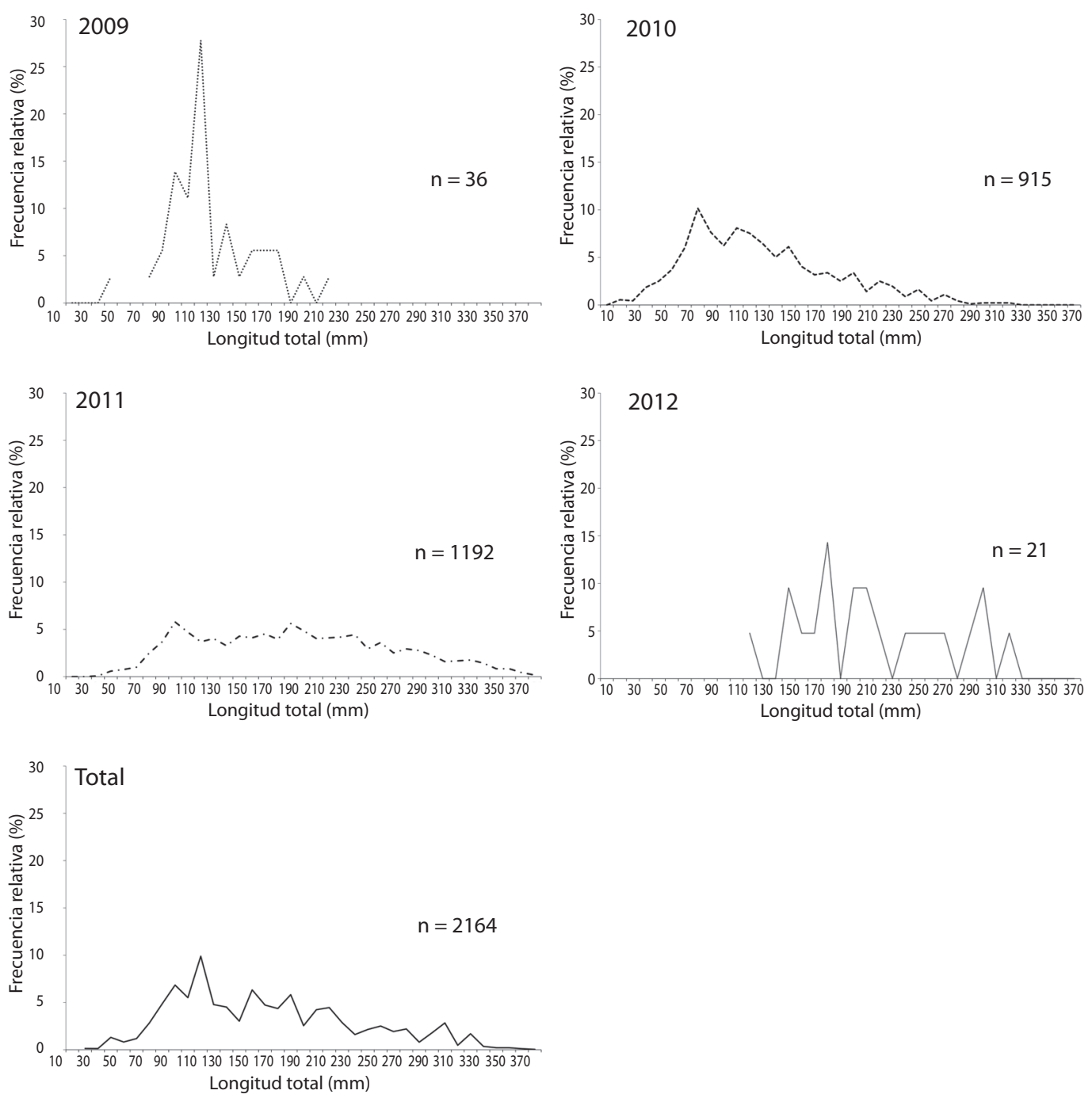

Fig. 2. Distribución de frecuencias relativas de las tallas del pez león (Pterois volitans) capturados durante cuatro años (2009, 2010, 2011, 2012 y total); en el Parque Nacional Arrecifes de Xcalak, Quintana Roo, México.

Fig. 2. Distribution of relative frequencies of the sizes of lionfish (Pterois volitans) captured during four years (2009, 2010, 2011, 2012 and total); in the Parque Nacional Arrecifes de Xcalak, Quintana Roo, Mexico.

una comparación de los valores obtenidos de esta ecuación con los de otros autores en varias localidades del Atlántico, mar Caribe y Golfo de México (Cuadro 2).

La estimación de la edad de los organismos al emplear la curva de crecimiento de Von Bertalanffy, reveló que en 2009, el $94 \%$ de los organismos capturados correspondieron al año 0 y un $6 \%$ al año 1 . Del 2010 al 2012 se presentaron cinco grupos de edad; el principal grupo en todos los años correspondió al de edad "0", indicativo de alto reclutamiento. Sin embargo, para 2011 y 2012 se obtuvieron porcentajes de $47 \%$ y $61 \%$ respectivamente, para edades entre uno y cinco años, lo que indicó un aumento en la proporción de organismos que han alcanzado la primera madurez sexual (190 mm $L t$ ). La longitud máxima recolectada en este estudio fue de $375 \mathrm{~mm}$ de $L t$, al realizar el retrocálculo de las edades, se estimó que este 


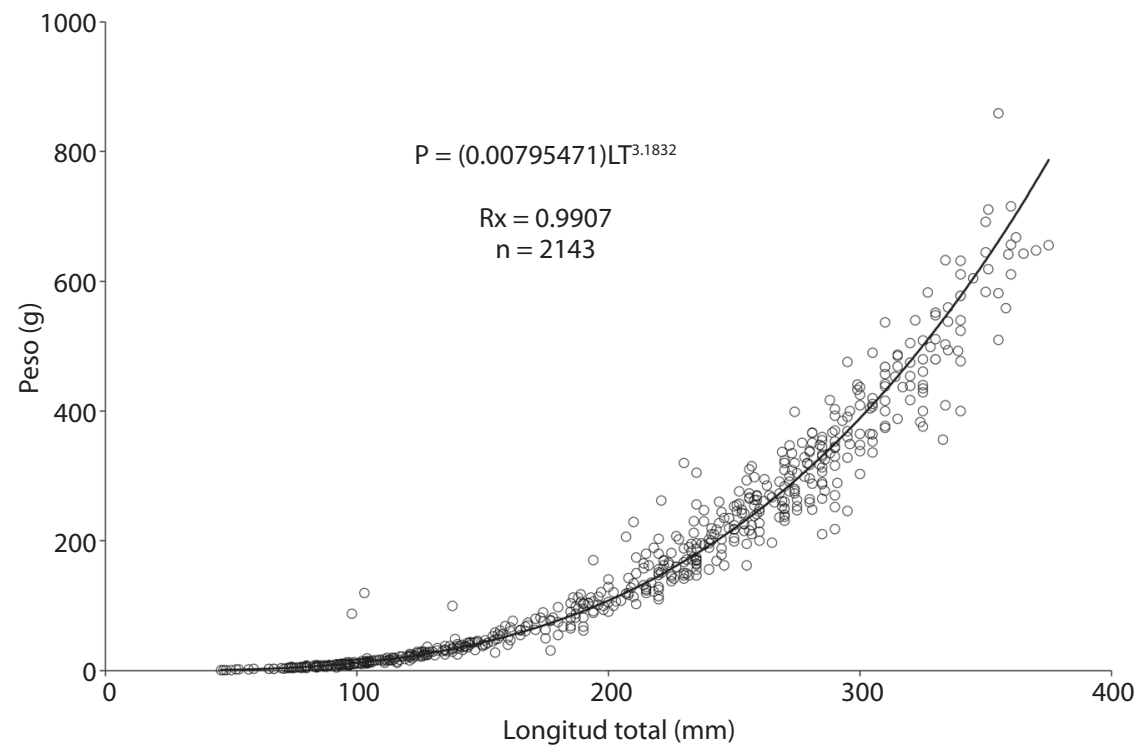

Fig. 3. Relación entre la longitud total y peso del pez león (Pterois volitans) capturados en el Parque Nacional Arrecifes de Xcalak, Quintana Roo, Caribe mexicano. La estimación de los valores fueron: $a(0.00795471), b(3.1832)$ y $R^{2}(0.9907)$. $\mathrm{P}=(0.00795471) \mathrm{LT}^{3.18}$

Fig. 3. Relation TL and Weight for lion fish (Pterois volitans) caught in the Parque Nacional Arrecifes de Xcalak, Quintana Roo, Mexican Caribbean. Values estimated a (0.00795471), b (3.1832) y R2 (0.9907). P=(0.00795471)LT $\mathrm{LT}^{3.18}$

\section{CUADRO 2}

Parámetros de la relación LT-Peso del pez león (Pterois volitans) en el noroeste del Océano Atlántico, Mar Caribe y Golfo de México

TABLE 2

Lion fish (Pterois volitans) Length-Weight (L-W) relationship at the northwest Atlantic Ocean, Caribbean Sea and Gulf of Mexico

\begin{tabular}{lcccc}
\multicolumn{1}{c}{ Localidad } & $a$ & $b$ & $r^{2}$ & Autor \\
Carolina del N, USA & 0.0000289 & 2.89 & - & Barbour et al., 2011 \\
Bahamas & 0.00497 & 3.2191 & - & Darling et al., 2011 \\
Puerto Rico & 0.08 & 3.11 & 0.958 & Toledo-Hernández et al., 2014 \\
Norte del Golfo de México & 0.00000207 & 3.34 & 0.98 & Dahl \& Patterson, 2014 \\
Little Cayman & 0.000003 & 3.24 & 0.97 & Edwards et al., 2014 \\
Costa Rica & 0.0235 & 2.81 & - & Sandel et al., 2015 \\
Xcalak, México & 0.00795471 & 3.1832 & 0.9907 & Este estudio \\
\hline
\end{tabular}

organismo presentaba una edad de cinco años. Este dato indicó que pudo haberse reclutado en el Caribe mexicano en el 2006; tres años antes del primer registro en 2009 (Fig. 4).

\section{DISCUSIÓN}

La distribución de tallas a lo largo del periodo de muestreo indica la presencia de juveniles y adultos, presumiéndose que la población de $P$. volitans se encuentra establecida en Xcalak.

Aunque el primer registro se realizó en enero de 2009, en los arrecifes de Cozumel (norte del Caribe mexicano) y en el sur (Xcalak y Banco Chinchorro), en julio del mismo año. Se presume que el flujo principal de transporte de larvas fue de sur a norte, 


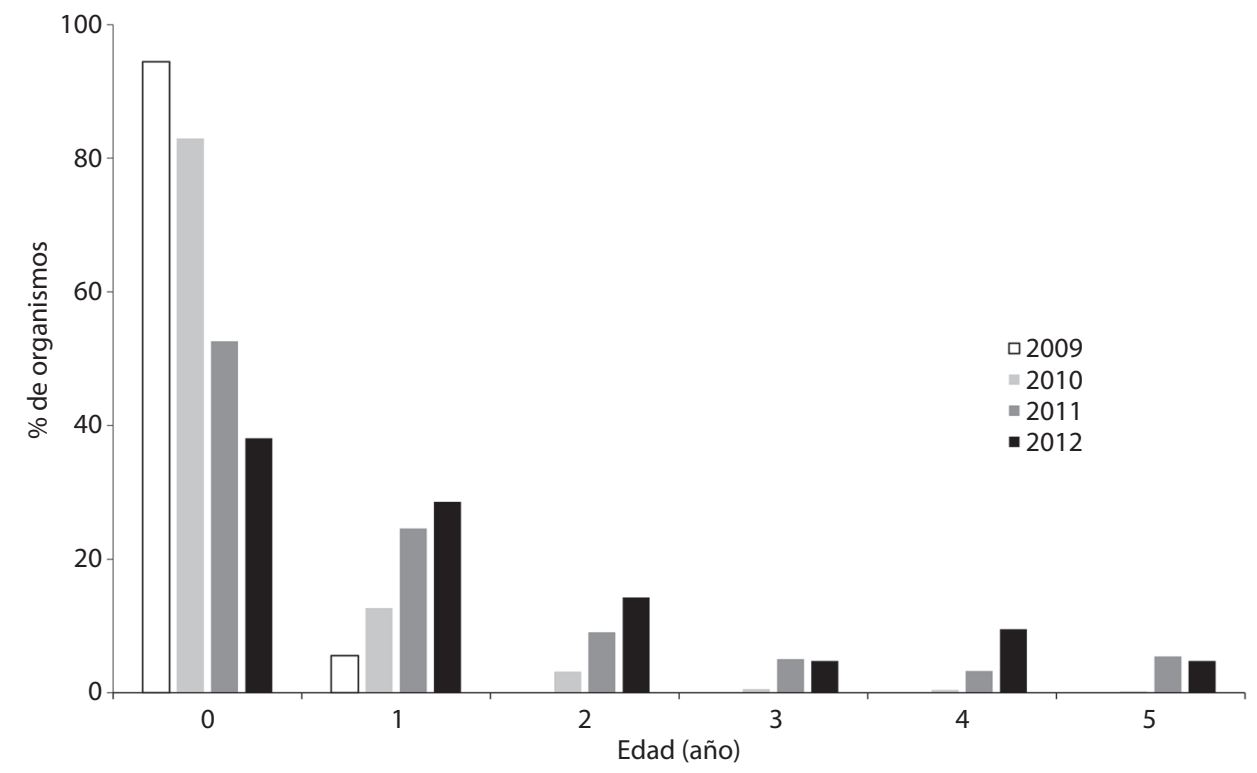

Fig. 4. Frecuencia de las clases de edad del pez león (Pterois volitans) para cada año de captura (2009, 2010, 2011 y 2012$).$ Fig. 4. Lionfish (Pterois volitans) age class frequency for each caught year (2009, 2010, 2011 and 2012).

debido a las corrientes oceánicas de la región (Vásquez-Yeomans et al., 2011); además de haberse reportado en diciembre 2008 en Belice (Schofield, 2009). Posiblemente, su primer registro en Cozumel puede atribuirse al alto número de buzos que frecuentan esta zona. Las tallas y pesos para Xcalak, se ubican dentro de los márgenes reportados por otros autores en varias zonas geográficas del Atlántico, Golfo de México, mar Caribe y en su área de distribución natural (Darling et al., 2011; Fogg et al., 2013; Toledo-Hernández et al., 2014).

Dentro de las etapas del proceso de invasión por especies exóticas, se encuentra la introducción al nuevo hábitat, la colonización inicial, el establecimiento exitoso y dispersión (Badii \& Landeros, 2007). Respecto a P. volitans, se ha observado que durante los primeros avistamientos en una determinada área geográfica, estos presentan tallas pequeñas, pudiendo ser indicativo de una invasión reciente, y que la población está conformada en su mayoría, por organismos en crecimiento (Claydon, Calosso, \& Traiger, 2012; Ruttenberg et al., 2012). Durante los siguientes años de invasión se ha constatado un aumento significativo en las longitudes promedio y tallas máximas (Dahl \& Patterson, 2014; Ruttenberg et al., 2012).

El aumento de tamaño se debe fundamentalmente al rápido crecimiento que presenta la especie, particularmente en las aguas tropicales del Caribe (Edwards et al., 2014); alcanzando tallas grandes como las observadas por Whitfield et al. (2007), en Carolina del Norte, y Darling et al. (2011) en el Caribe.

Las tallas registradas en Carolina del Norte son superiores $(450 \mathrm{~mm} L t)$ a las halladas en este estudio (Whitfield et al., 2007); esto puede deberse a la diferencia en el tiempo de invasión de las costas de Estados Unidos a partir del 2000 y el Caribe mexicano hasta 2009 (Schofield, 2009). Cabe hacer mención que el último mes de muestreo de este trabajo (febrero 2012), se encontró el pez de mayor tamaño $(375 \mathrm{~mm}$ $L t$ ). Sin embargo, posteriormente se han reportado capturas con tallas superiores a $380 \mathrm{~mm}$ Lt (Hernández-Millán, com. pers) y para agosto 2012, la talla máxima encontrada para Banco Chinchorro, México fue de $415 \mathrm{~mm} \mathrm{Lt}$ (Obs. Pers). Esto puede servir como referencia para 
inferir que en el transcurso de los próximos años se podrán encontrar tallas superiores a las reportadas en este estudio.

Debido a que la invasión es reciente, no se tenía reporte de la relación de la $L t$-Peso en el Caribe mexicano, por lo que el presente trabajo proporciona la información básica que puede conllevar a entender la dinámica de la población de $P$. volitans en los arrecifes del sur de México. El efecto de alometría demostrado en el presente estudio, muestra un acelerado crecimiento en la longitud al inicio, en una etapa avanzada de su ciclo disminuye y el pez continúa ganando peso. Esto puede variar entre los organismos y estar relacionado con el tamaño de la muestra, época del año, alimentación, estado de madurez, factores ambientales $\mathrm{y}$ con el rápido crecimiento en las primeras etapas de vida (Sandel, Martínez-Fernández, Wangpraseurt, \& Sierra, 2015; Toledo-Hernández et al., 2014).

Para estimar la edad de los organismos, se tomaron como referencia los parámetros obtenidos por Edwards et al. (2014), infiriendo que la población de Xcalak es similar a la de Little Cayman, debido a que ambas se encuentran en la región del Caribe.

Aunque la primera captura se registró en 2009, el retrocálculo de las longitudes obtenidas; se puede inferir que $P$. volitans estaba presente aproximadamente desde el 2006, es decir, tres años antes del primer reporte. Probablemente, en densidades poblacionales muy bajas, motivo por el cual no fue detectado en el momento (Claydon et al., 2012; Fogg et al., 2013).

Debido al rápido crecimiento en la etapa juvenil, el pez león alcanza la etapa reproductiva en tan solo un año (Barbour, Allen, Frazer, \& Sherman, 2011). Claydon et al. (2012), estimaron que el $71 \%$ de los peces que se encontraban en los hábitats de poca profundidad tenían menos de un año. Para Xcalak a partir del 2011, se presentó mayor porcentaje de organismos con edades superiores al año, es decir tallas de aproximadamente $190 \mathrm{~mm}$ de $L t$, las cuales corresponden a organismos reproductores (Gardner et al., 2015).
Los resultados obtenidos permiten un mejor entendimiento del establecimiento de esta especie y su dinámica poblacional ya que en tan solo cuatro años, ha logrado invadir y crecer exitosamente.

Dada la aparición súbita en todo el Caribe de las poblaciones de $P$. volitans y su reconocida capacidad invasora, parecen estar tomadas las medidas de control como la promoción de captura a través de torneos de pesca, su comercialización local y realización de talleres informativos (Gómez-Lozano et al., 2013). Un monitoreo continuo de la población así como sus relaciones ecológicas con el ambiente y presas nativas, serán necesarios para esclarecer el impacto real sobre los ecosistemas y así proponer las medidas de control más adecuadas en las costas mexicanas.

\section{AGRADECIMIENTOS}

A cada uno de los pescadores y pobladores de Xcalak, que contribuyeron en las capturas; a los guardaparques del PNAX que de diferentes maneras contribuyen al control de esta especie, en especial a la bióloga Nallely Hernández, Martha Hernández y Wendy Chi. A Holger Weissenberger por la elaboración del mapa. A la Comisión Nacional de Áreas Naturales Protegidas (CONANP), por permitir la estancia en el PNAX, al Instituto Tecnológico de Chetumal y Ecosur-Unidad Chetumal.

\section{RESUMEN}

El pez león ( $P$. volitans), ha invadido todo el Caribe mexicano y Golfo de México, con un potencial de causar impactos negativos en el arrecife. En el sur del Caribe Mexicano se registró por vez primera en julio 2009, y a seis años del primer reporte, se han implementado algunas medidas de control como torneos de pesca y su comercialización local. No obstante, la información sobre su biología e inicio de invasión aún es escasa, por lo que este estudio analizó la estructura poblacional de 2164 organismos recolectados entre 2009 y 2012, un incremento en las tallas para cada año fue observado, con promedios de Longitud Total $(L t): 118 \pm 34.8,133 \pm 56.3,187 \pm 74.8$ y $219 \pm 72.4 \mathrm{~mm}$ respectivamente. El establecimiento del pez león en el área de estudio es demostrado por la presencia de tallas juveniles $(20 \mathrm{~mm} \mathrm{Lt}$ ) hasta organismos de $375 \mathrm{~mm} \mathrm{Lt}$. Al realizar 
el retrocálculo de las longitudes obtenidas se estimó que el organismo de mayor talla pudo reclutarse a principios del 2006, tres años antes del primer reporte. Un monitoreo continuo de la población así como un estudio ecológico, nos permitirá dilucidar el impacto real en los ecosistemas de la región y así proponer las medidas de control más efectivas.

Palabras clave: pez león, especie invasora, Caribe mexicano, estructura de tallas.

\section{REFERENCIAS}

Aguilar-Perera, A., \& Tuz-Sulub, A. (2010). Non-native, invasive red lionfish (Pterois volitans [Linnaeus, 1758]: Scorpaenidae), is first recorded in the southern Gulf of Mexico, off the northern Yucatan Peninsula, Mexico. Aquatic Invasions, 5, 9-12.

Albins, M. A. (2013). Effects of invasive Pacific red lionfish Pterois volitans versus a native predator on Bahamian coral-reef fish communities. Biological Invasions, 15(1), 29-43.

Albins, M. A., \& Hixon, M. A. (2008). Invasive IndoPacific lionfish Pterois volitans reduce recruitment of Atlantic coral-reef fishes. Marine Ecology Progress Series, 367, 233-238.

Albins, M. A., \& Hixon, M. A. (2013). Worst case scenario: Potential long-term effects of invasive predatory lionfish (Pterois volitans) on Atlantic and Caribbean coral-reef communities. Environmental Biology of Fishes, 96, 1151-1157.

Badii, M. H. \& Landeros, J. (2007). Invasión de especies o el tercer jinete de apocalipsis ambiental, una amenaza a la sustentabilidad. International Journal of Good Conscience, 2(1), 39-53.

Barbour, A. B., Allen, M. S., Frazer, T. K., \& Sherman, K. D. (2011). Evaluating the potential efficacy of invasive lionfish (Pterois volitans) removals. PLoS ONE, 6(5), 1-7.

Claydon, J. A. B., Calosso, M. C., \& Traiger, S. B. (2012). Progression of invasive lionfish in seagrass, mangrove and reef habitats. Marine Ecology Progress Series, 448, 119-129.

CONANP. (2004). Programa de Manejo Parque Nacional, Arrecifes de Xcalak. Diario Oficial de La Federación.

Dahl, K. A., \& Patterson, W. F. (2014). Habitat-specific density and diet of rapidly expanding invasive red lionfish, Pterois volitans, populations in the Northern Gulf of Mexico. PLoS ONE, 9(8), e105852.

Darling, E. S., Green, S. J., O’Leary, J. K., \& Côté, I. M. (2011). Indo-Pacific lionfish are larger and more abundant on invaded reefs: A comparison of Kenyan and Bahamian lionfish populations. Biological Invasions, 13, 2045-2051.
Edelist, D., Rilov, G., Golani, D., Carlton, J. T., \& Spanier, E. (2013). Restructuring the Sea: Profound shifts in the world's most invaded marine ecosystem. Diversity and Distributions, 19, 69-77.

Edwards, M. A., Frazer, T. K., \& Jacoby, C. A. (2014). Age and growth of invasive lionfish (Pterois spp.) in the Caribbean Sea, with implications for management. Bulletin of Marine Science, 90(4), 953-966.

Fogg, A. Q., Hoffmayer, E. R., Driggers III, W. B. D., Campbell, M. D., Pellegrin, G. J., \& Stein, W. (2013). Distribution and Length Frequency of Invasive Lionfish (Pterois sp.) in the Northern Gulf of Mexico. Gulf and Caribbean research, 25, 111-115.

Gardner, P. G., Frazer, T. K., Jacoby, C. A., Roy, P., Yanong, E., Frank, W., \& Iii, P. (2015). Reproductive biology of invasive lionfish (Pterois spp.). Frontiers in Marine Science, 2(7), 1-10.

Gómez-Lozano, R., Anderson, L., Akins, J. L., Buddo, D. S. A., García-Moliner, G., Gourdin, F., Laurent, M., Lilyestrom, C., Morris, J. A. Jr., Ramnanan, N., \& Torres, R. (2013). Estrategia regional para el control del Pez León invasor en el Gran Caribe. Iniciativa Internacional sobre los Arrecifes Coralinos.

Green, S. J., Akins, J. L., Maljković, A., \& Côté, I. M. (2012). Invasive lionfish drive Atlantic coral reef fish declines. PLoS ONE, 7.

IMTA, Conabio, GECI, Aridamérica, \& TNC. (2007). Especies invasoras de alto impacto a la biodiversidad. Prioridades en México. Jiutepec, Morelos.

Kramer, P. A, \& Kramer, P. R. (2002). Ecoregional conservation planning for the Mesoamerican Caribbean Reef (M. McField, Ed.). Washington, D.C. World Wildlife Fund.

Ricker, W. E. (2010). Computation and interpretation of biological statistics of fish populations. Bulletin Fishery Resources Canadian (Vol. 191).

Ruttenberg, B. I., Schofield, P., Akins, J. L., Acosta, A., Feeley, M. W., Blondeau, J., Smith, S. G., \& Ault, J. S. (2012). Rapid invasion of Indo-Pacific lionfishes (Pterois volitans and Pterois miles) in the Florida Keys, USA: Evidence from multiple pre- and postinvasion data sets. Bulletin of Marine Science, 88(4), 1051-1059.

Sandel, V., Martínez-Fernández, D., Wangpraseurt, D., \& Sierra, L. (2015). Ecology and management of the invasive lionfish Pterois volitans/miles complex (Perciformes: Scorpaenidae ) in Southern Costa Rica. Revista de Biología Tropical, 63(1), 213-221.

Santander-Monsalvo, J., López-Huerta, I., Aguilar-Perera, A., \& Tuz-Sulub, A. (2012). First record of the red lionfish (Pterois volitans [Linnaeus, 1758]) off the coast of Veracruz, Mexico. BioInvasions Records, 1(2), 121-124. 
Schofield, P. J. (2009). Geographic extent and chronology of the invasion of non-native lionfish (Pterois volitans [Linnaeus 1758] and P. miles [Bennett 1828]) in the Western North Atlantic and Caribbean Sea. Aquatic Invasions, 4(3), 473-479.

Schofield, P. J. (2010). Update on geographic spread of invasive lionfishes (Pterois volitans [Linnaeus, 1758] and P. miles [Bennett, 1828]) in the Western North Atlantic Ocean, Caribbean Sea and Gulf of Mexico. Aquatic Invasions, 5, 117-122.

Schultz, E. T. (1986). Pterois volitans and Pterois miles: Two Valid Species. Copeia, 1986, 686.

Semmens, B. X., Buhle, E. R., Salomon, A. K., \& Pattengill-Semmens, C. V. (2004). A hotspot of non-native marine fishes: Evidence for the aquarium trade as an invasion pathway. Marine Ecology Progress Series, 266, 239-244.

Sutherland, W. J., Clout, M., Côté, I. M., Daszak, P., Depledge, M. H., Fellman, L., Fleishman, E., Gibbons, D., Impey, A., Lawton, J., Lickorish, F., Lindenmayer, D., Lovejoy, T., Mac Nally, R., Madgwick, J., Peck L., Pretty, J., Prior, S., Redford, K., Scharlemann, J., Spalding, M., \& Watkinson, A. R. (2010). A horizon scan of global conservation issues for 2010 . Trends in Ecology and Evolution, 25, 1-7.
Toledo-Hernández, C., Vélez-Zuazo, X., Ruiz-Diaz, C. P., Patricio, A. R., Mège, P., Navarro, M., Sabat, A. M., Betancur, R. R., \& Papa, R. (2014). Population ecology and genetics of the invasive lionfish in Puerto Rico. Aquatic Invasions, 9, 227-237.

Valdez-Moreno, M., Quintal-Lizama, C., Gómez-Lozano, R., \& García-Rivas, M. D. C. (2012). Monitoring an alien invasion: DNA barcoding and the identification of lionfish and their prey on coral reefs of the Mexican Caribbean. PLoS ONE, 7(6), 1-8.

Vásquez-Yeomans, L., Carrillo, L., Morales, S., Malca, E., Morris, J. A., Schultz, T., \& Lamkin, J. T. (2011). First larval record of Pterois volitans (Pisces: Scorpaenidae) collected from the ichthyoplankton in the Atlantic. Biological Invasions, 13, 2635-2640.

Von Bertalanffy, L. (1957). Quantitative laws in metabolism and growth. The Quarterly Review of Biology, $32,217-231$.

Whitfield, P. E., Hare, J. A., David, A. W., Harter, S. L., Muñoz, R. C., \& Addison, C. M. (2007). Abundance estimates of the Indo-Pacific lionfish Pterois volitans/ miles complex in the Western North Atlantic. Biological Invasions, 9, 53-64. 